\title{
Role of freezing and fear in avoidance decrements following mammillothalamic tractotomy in cat: II. Appetitively motivated behavior'
}

E. E. KRIECKHAUS

POULTRY HUSBANDRY DEPARTMENT, UNIVERSITY OF CALIFORNIA AT DAVIS

Although previous work (Krieckhaus, 1964a; Krieckhaus $\mathcal{E}$ Chi, 1966) suggests that it is unlikely that the avoidance decrements following lesions of the mammillothalamic tract (MTT) are attributable to increased freezing behavior, in each case evaluation of freezing was confounded with concomitant changes in avoidance behavior. In the present experiment, freezing following MTT lesions was evaluated independently of avoidance behavior by using either the conditioned emotional response (CER) or an appetitively motivated visual discrimination in which the incorrect response was punished. In neither case was there any indication of increases in freezing or other signs of fear following lesions of the MTT. We thus conclude that the reported increases in freezing behavior following MTT lesions are a result of rather than a cause of a primary decrement in avoidance behavior.

It is now well established that in cats retention of a two-way (shuttle) conditioned avoidance response (CAR) is severely reduced following lesions of the mammillothalamic tract (Thomas, Fry, Fry, Slotnick, \& Krieckhaus, 1963; Krieckhaus, 1964a; Krieckhaus \& Chi, 1966). It had appeared earlier (Thomas et al, 1963) that this avoidance deficit might be attributable to increased freezing behavior. Although this explanation has received little support in subsequent work (Krieckhaus, 1964a; Krieckhaus \& Chi, 1966), these studies can not be considered conclusive since in each case evaluation of changes in freezing was confounded with concomitant changes in avoidance behavior.

The present experiment is intended to evaluate the effect of MTT lesions on fear and freezing behavior in a non-avoidance situation. This was accomplished by using: (1) the conditioned emotional response (CER), a measure which appears to have considerable construct validity in assessing effects of limbic system lesions on fear and freezing (see discussion by Kenyon \& Krieckhaus, 1965), and (2) two appetitively motivated visual discrimination responses, on one of which the incorrect response was punished by shock.

Method

The apparatus used for the visual discrimination-a modified Wisconsin general testing apparatus (WGTA)has been described by Thomas et al (1963) and the apparatus for the CER-a cat Skinner box-has been described by Krieckhaus (1964b). Seven naive, mongrel, female cats served as Ss. Five cats were trained on the CER and the two visual discriminations. One cat (No. 796) was trained on the visual discrimination without punishment, and on a two-way (shuttle) avoidance response. Results for this cat on the later task have already been reported in a prior study (Krieckhaus, 1964a). The last cat (No. 752) was trained on the CER, both visual discriminations and the two-way CAR. Each cat was taught its respective habits in the order mentioned above.

For the CER each cat was first trained to press the lever for milk with a shaping procedure. When the response rate had stabilized the cat was switched to a VI $30 \mathrm{sec}$. schedule with a 5 sec. limited hold. After the lever pressing rate had again stabilized, the CER was established. Several minutes after the cat began its daily session of lever pressing for milk a CS (attenuated sound of a buzzer) was turned on for $2 \mathrm{~min}$. A few days later when the cat no longer showed a response to this $\mathrm{CS}$, a $1.0 \mathrm{ma}$ foot shock of $2 \mathrm{sec}$. duration was given following termination of the CS.

Following CER training the cat was taught the appetitively motivated visual discrimination response described by Thomas et al (1963). Training procedures were the same except that the cats were taught two discriminations. On the discrimination learned first an incorrect response was simply not rewarded with food: this was the procedure used by Thomas et al. On the second discrimination, the incorrect response was also punished with mild foot shock (.5-1.5 ma) delivered through the grid floor. This shock initially elicited freezing and general disruption of ongoing behavior, with the result that many hundreds of trials were necessary to establish both visual discrimination responses.

Following training, electrolytic lesions were stereotaxically aimed bilaterally at the MTT. Lesioning and histological procedures have been described by Krieckhaus (1964a). Following a 2 week postoperative recovery period, each cat was tested under preoperative conditions except that no shock was administered.

\section{Results}

Of the six cats trained on the CER and both visual discriminations, in one the MTT was destroyed completely bilaterally, in two the MTT was completely destroyed on one side and partially destroyed on the other side, and in the remaining three cats the MTT was completely spared. In all cats there was no change in lever pressing for milk or in either of the visual discrimination tasks. Furthermore, cat No. 796, which sustained a complete MTT lesion on one side and a 

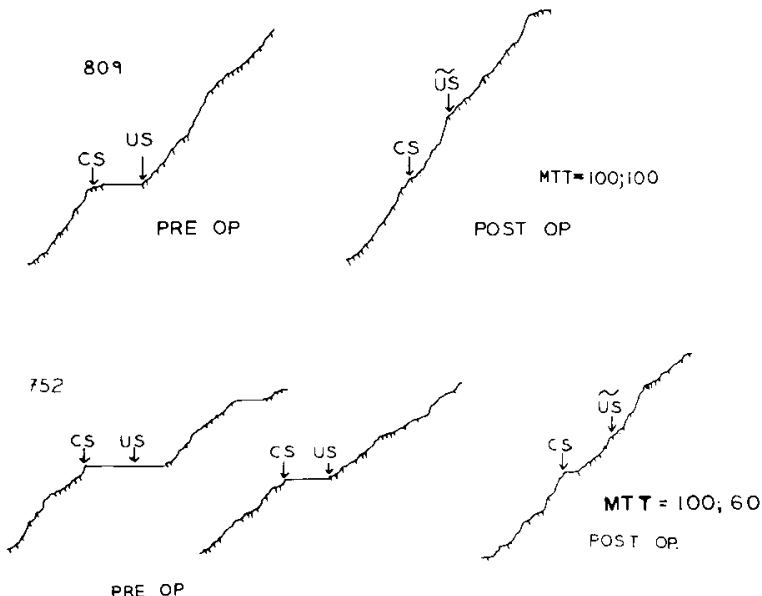

PRE OP
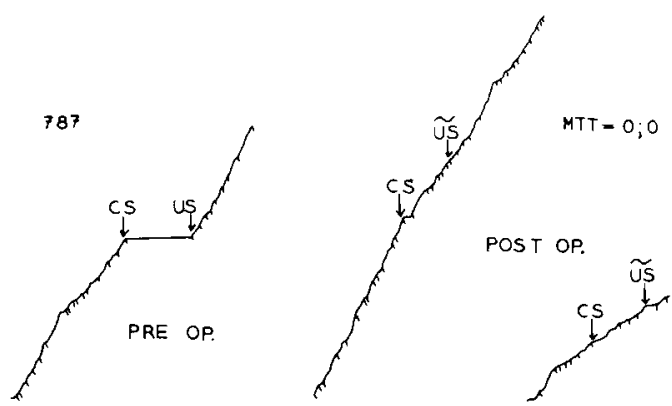

Fig. 1. CER, pre- and postoperative.

partial MTT lesion on the other side and which showed a complete loss of the two-way conditioned avoidance response (Krieckhaus, 1964a) showed perfect retention of this visual discrimination task.

In the case of the CER all six cats lost their CER within the first few days of postoperative testing. In the two cats with the greatest MTT damage and in one cat in which the MTT was undamaged it is clear from Fig. 1 that there was an almost complete loss of the CER from the first postoperative test day. Furthermore, cat No. 752, which had also been trained on the two-way CAR with the same CS used in CER training, failed to cross the hurdle within the CS-US interval on more than half the avoidance trials and required seven shocks to relearn. Clearly one cannot attribute the CAR decrement to increases in fear or freezing elicited by the CS, since the cat showed a complete absence of a CER to the same CS.

\section{Discussion}

There now exists considerable data which indicate that the decrements in avoidance behavior following MTT transection are not attributable simply to increases in fear or its manifestations. First, Krieckhaus (1964a) showed in cats with partial MTT destruction and thus less severe avoidance decrements, that any increase in freezing was noticeable only after the animal had failed to avoid on several trials, and in some cases there was never any noticeable increase in freezing. Second, in the same study the two-way CAR was punished by shocking the cat for crossing the hurdle. Each cat soon learned to stop crossing, and the hurdle jump response was replaced by an intense freezing reaction. There was no tendency for the animals with MTT lesions to stop crossing any sooner than controls. Thus, a change in behavior that presumably would have been facilitated by increased freezing was not enhanced by MTT lesions. Third, Krieckhaus \& Chi (1966) have shown that if a stimulus capable of producing intense fear and freezing is introduced along with the regular CS, the animal's avoidance performance is actually enhanced. Fourth, in this same study, when cats were tested in a two-way avoidance apparatus large enough for the cat to move around in if it did not cross the hurdle, CAR decrements after MTT lesions were often accompanied by behavior quite incompatible with freezing. Finally, in the present study, independent of any confounding with changes in avoidance behavior, there was no increase in either the CER or in the effect of fear in disrupting a difficult visual discrimination task following MTT lesions.

We thus conclude that the increase in freezing behavior that has been reported following lesions of the MTT is probably a result, not the cause, of a primary avoidance decrement. This contention is supported by an incidental observation that preoperatively, in the normal cat, marked increases in freezing and other manifestations of fear can be reliably produced by prohibiting the cats from executing the avoidance response. In the two-way CAR this was done by not raising the door separating the two compartments when the CS was presented. In a lever press CAR, the results of which will be reported later, this was accomplished by retracting the lever when the CS came on.

\section{References}

Kenyon, J., \& Krieckhaus, E. E. Decrements in one-way avoidance learning following septal lesions in rats. Psychon. Sci., 1965, 3, 113-114

Krieckhaus, E. E. Decrements in avoidance behavior following mammillothalamic tractotomy in cats. J. Neurophysiol., 1964a, 27, 753-767.

Krieckhaus, E. E. Ultrasound and psychology. In D. Gordon (Ed.), Ultrasound as a diagnostic and surgical tool. Edinburgh: Livingston, $1964 \mathrm{~b}$.

Krieckhaus, E. E., \& Chi, C. C. Role of freezing and fear in avoidance decrements following mammillothalamic tractotomy in cat. I. Two-way avoidance behavior. Psychon. Sci., 1966, 4, 263-264.

Thomas, G. J., Fry, W. J., Fry, F. J., Slotnick, B. M., \& Krieckhaus, E. E. Behavioral effects of mammillothalamic tractotomy in cats. J. Neurophysiol., 1963, 26, 857-876.

\section{Note}

1. This research was supported by Grant 10885 from the National Science Foundation to the Biophysical Research Laboratory, University of Illinois. 